\title{
Global Solutions of IBVP to Nonlinear Equation of Suspended String
}

\author{
Jaipong WONGSAWASDI and Masaru YAMAGUCHI
}

Tokai University

(Communicated by M. Tanaka)

\begin{abstract}
We shall consider IBVP to a nonlinear equation of suspended string with uniform density to which a nonlinear time-independent outer force works. We shall show the existence of time-global weak solutions of IBVP. To prove our result we shall use the function spaces defined by [Ya1], and apply the method due to Sattinger [Sat] based on the potential-well and the Galerkin method.
\end{abstract}

\section{Introduction}

Let $\Omega$ be a cylindrical domain $(0, a) \times(0, T)$. Consider IBVP for a nonlinear equation of a flexible and heavy suspended string of finite length $a$ with uniform density

$$
\left\{\begin{array}{l}
\partial_{t}^{2} u(x, t)+L u(x, t)+f(x, u(x, t))=0, \quad(x, t) \in \Omega, \\
u(a, t)=0, \quad t \in(0, T), \\
u(x, 0)=\phi(x), \quad \partial_{t} u(x, 0)=\psi(x), \quad x \in(0, a),
\end{array}\right.
$$

where $L=L_{0}\left(x, \partial_{x}\right)$ is a second order differential operator of the form

$$
L=-\left(x \partial_{x}^{2}+\partial_{x}\right)
$$

and $f(x, u)$ is of $C^{1}$ and monotone decreasing, and has the zero point of $p$-order at $u=0$ $(p>1)$. The exact assumption on $f$ will be given as (A) in section 3 . Clearly $u \equiv 0$ is a steady state solution of the equation. For the derivation of the equation of the suspended string, see Koshlyakov-Gliner-Smirnov [K-G-S] and Korenev [Ko]. See also Yamaguchi [Ya1].

[K-G-S], [Ko] and [Ya1] deal with IBVP to linear equations of suspended string with a power density $x^{\mu}, \mu>-1$ :

$$
\left\{\begin{array}{l}
\partial_{t}^{2} u+L_{\mu} u+f(x, t)=0, \quad(x, t) \in(0, a) \times R_{t}^{1}, \\
u(a, t)=0, \quad t \in R_{t}^{1}, \\
u(x, 0)=\phi(x), \quad \partial_{t} u(x, 0)=\psi(x), \quad x \in(0, a),
\end{array}\right.
$$

Received July 11, 2006; revised October 13, 2006

This research is supported in part by Grant-in-Aid for Scientific Research (C)(2) No.15540213, Japan Society for the Promotion of Science. 
where $L_{\mu}$ is a second order differential operator of the form

$$
L_{\mu}=-\left(\frac{x}{\mu+1} \partial_{x}^{2}+\partial_{x}\right)
$$

[K-G-S] and [Ko] studied the case where $\mu>-1$ and $f$ is a time-periodic single mode of the form $f(x, t)=A \sin \omega t J_{0}\left(\mu_{k} \sqrt{x / a}\right)$, where $J_{0}$ is the 0 -order Bessel function and $\mu_{k}$ is a zero point of $J_{0}$. [Ya1] treated the case where $\mu \geq 0$ and $f$ is time quasiperiodic. They showed that (LP) has a unique time-global solution which is represented by the Fourier series. In [K-G-S] and [Ko] the solutions are formal in the sense that the convergence of the Fourier series is not shown. In [Ya1] the solutions belong to some suitable weighted Sobolev type function spaces so that every solution is classical. In [Ya1] it is also shown that every solution of (LP) is almost periodic in $t$ under the Diophantine conditions. Besides the almost periodic structure of solutions is clarified.

On the other hand, it seems to authors that there have been no researches on IBVP when the equation is nonlinear. The purpose of this paper is to show that nonlinear IBVP (P) has a time-global solution that lies in the potential well around the origin. It follows that the energy of every solution is bounded in $t \in(0, T)$. In this paper we shall treat weak solutions (the definition will be given in section 3 ).

In order to show our result, first we shall introduce function spaces defined by [Ya1] and use properties like compact or continuous embedding and estimates in the spaces like a generalized Sobolev inequality, a generalized Poincaré inequality. Secondly we shall apply the method due to Sattinger [Sat] to $(\mathrm{P})$ based on the potential well and the Galerkin method. [Sat] developed his method to show the existence of global solutions of IBVP to nonlinear wave equations. See also [L].

To apply a generalized Sobolev inequality to our problem, we need to restrict ourselves to a uniform density $\rho(x)=\alpha x^{0}$, i.e., $\mu=0$.

For the existence of infinitely many time-periodic solutions of nonlinear equations, see [Ya2]. See also [Ya-Na-Ma] for the periodic problem to nonlinear damped equations with periodic forcing term.

This paper will be organized as follows. In section 2 we shall give notation and definitions of function spaces, and some properties of the function spaces and inequalities used in later sections. Also a brief sketch to the eigenvalue problem for $L_{\mu}$ will be given. In section 3 our theorem will be formulated. We shall define the potential well corresponding to $(\mathrm{P})$ and study its properties. In section 4 using the Galerkin method and the compactness method, the theorem will be proved.

\section{Function Spaces, Operator $L$, the Basic Inequalities}

Let $R_{+}^{1}$ and $Z_{+}$be the set of nonnegative numbers and nonnegative integers, respectively. In the following we let $\mu \geqq 0$, and all the functions are real-valued. 
2.1. Definitions of Function Spaces. Let $O$ be any open set in $R^{n}$. Let $p \geqq 1$ and $s \in Z_{+} . L^{p}(O)$ and $H^{s}(O)$ are the usual Lebesgue and Sobolev spaces, respectively.

We shall set function spaces defined in [Ya1]. $L^{p}\left(0, a ; x^{\mu}\right)$ is a Banach space whose elements $f(x)$ are measurable in $(0, a)$ and satisfy $x^{\mu / p} f(x) \in L^{p}(0, a)$, where the norm is defined by

$$
|f|_{L^{p}\left(0, a ; x^{\mu}\right)}=\left(\int_{0}^{a} x^{\mu}|f(x)|^{p} d x\right)^{1 / p} .
$$

Clearly $L^{p}\left(0, a ; x^{0}\right)=L^{p}(0, a) . L^{2}\left(0, a ; x^{\mu}\right)$ is a Hilbert space with the inner product defined by

$$
(f, g)_{L^{2}\left(0, a ; x^{\mu}\right)}=\int_{0}^{a} x^{\mu} f(x) g(x) d x .
$$

$H^{s}\left(0, a ; x^{\mu}\right)$ is a Hilbert space whose elements $f$ and their weighted derivatives $x^{j / 2} f^{(j)}, j=1, \ldots, s$, belong to $L^{2}\left(0, a ; x^{\mu}\right)$, where $f^{(j)}$ means the $j$-th derivative of $f$. Its norm is defined by

$$
\begin{aligned}
|f|_{H^{s}\left(0, a ; x^{\mu}\right)} & =\left(\sum_{j=0}^{s}\left|x^{j / 2} f^{(j)}\right|_{L^{2}\left(0, a ; x^{\mu}\right)}^{2}\right)^{1 / 2} \\
& =\left(\sum_{j=0}^{s} \int_{0}^{a} x^{\mu+j} f^{(j)}(x)^{2} d x\right)^{1 / 2} .
\end{aligned}
$$

Let $T>0$. Let $\Omega=(0, a) \times(0, T) . L^{p}\left(\Omega ; x^{\mu}\right)$ is a Banach space whose elements $f(x, t)$ are measurable in $\Omega$ and satisfy $x^{\mu / p} f(x, t) \in L^{p}(\Omega)$, where the norm is defined by

$$
|f|_{L^{p}\left(\Omega ; x^{\mu}\right)}=\left(\int_{\Omega} x^{\mu}|f(x, t)|^{p} d x d t\right)^{1 / p} .
$$

In particular, $L^{p}\left(\Omega ; x^{0}\right)=L^{p}(\Omega)$ holds.

$H^{s}\left(\Omega ; x^{\mu}\right)$ is a Hilbert space whose elements $f$ and their weighted derivatives $x^{j / 2} \partial_{x}^{j} \partial_{t}^{k} f, 0 \leqq j+k \leqq s$, belong to $L^{2}\left(\Omega ; x^{\mu}\right)$. Its norm is defined by

$$
\begin{aligned}
|f|_{H^{s}\left(\Omega ; x^{\mu}\right)} & =\left(\sum_{j+k \leqq s}\left|x^{j / 2} \partial_{x}^{j} \partial_{t}^{k} f\right|_{L^{2}\left(\Omega ; x^{\mu}\right)}^{2}\right)^{1 / 2} \\
& =\left(\sum_{j+k \leqq s} \int_{\Omega} x^{\mu+j}\left(\partial_{x}^{j} \partial_{t}^{k} f(x, t)\right)^{2} d x d t\right)^{1 / 2} .
\end{aligned}
$$

$H_{0}^{1}\left(0, a ; x^{\mu}\right)$ is a subspace as Hilbert space of $H^{1}\left(0, a ; x^{\mu}\right)$ whose elements $f$ satisfy $f(a)=0$. Also $H_{0}^{1}\left(\Omega ; x^{\mu}\right)$ is a subspace of $H^{1}\left(\Omega ; x^{\mu}\right)$ whose elements $f$ satisfy $f(a, t)=$ 
0 for almost all $t . \quad K^{s}\left(0, a ; x^{\mu}\right)$ is a subspace of $H^{s}\left(0, a ; x^{\mu}\right)$ whose elements $f$ satisfy $L_{\mu}^{j} f \in H_{0}^{1}\left(0, a ; x^{\mu}\right)$ for $j=0, \ldots,[(s-1) / 2]$. Note that

$$
\begin{gathered}
K^{0}\left(0, a ; x^{\mu}\right)=L^{2}\left(0, a ; x^{\mu}\right), \quad K^{1}\left(0, a ; x^{\mu}\right)=H_{0}^{1}\left(0, a ; x^{\mu}\right), \\
K^{2}\left(0, a ; x^{\mu}\right)=H^{2}\left(0, a ; x^{\mu}\right) \cap H_{0}^{1}\left(0, a ; x^{\mu}\right) .
\end{gathered}
$$

PROPOSITION 2.1 ([Ya1]). $L_{\mu}$ has the following properties:

(i) For $f \in K^{2}\left(0, a ; x^{\mu}\right)$ and $g \in K^{1}\left(0, a ; x^{\mu}\right)$,

$$
\left(L_{\mu} f, g\right)_{L^{2}\left(0, a ; x^{\mu}\right)}=\int_{0}^{a} \frac{x^{\mu+1}}{\mu+1} \partial_{x} f(x) \partial_{x} g(x) d x .
$$

(ii) $L_{\mu}$ is a positive definite self-adjoint elliptic operator in $L^{2}\left(0, a ; x^{\mu}\right)$ with domain $D(L)=K^{2}\left(0, a ; x^{\mu}\right)$, i.e.

$$
\left(L_{\mu} f, f\right)_{L^{2}\left(0, a ; x^{\mu}\right)} \geqq 0, \quad\left(L_{\mu} f, g\right)_{L^{2}\left(0, a ; x^{\mu}\right)}=\left(f, L_{\mu} g\right)_{L^{2}\left(0, a ; x^{\mu}\right)}
$$

hold for $f, g \in K^{2}\left(0, a ; x^{\mu}\right)$.

PROPOSITION 2.2. The following holds:

(i) If $s \geqq 1, H^{s}\left(0, a ; x^{\mu}\right)$ is embedded continuously in $L^{2}\left(0, a ; x^{\mu-\delta}\right)$ for any $\delta$ with $\delta \leqq 1$ and $\delta<\mu+1$.

(ii) If $s \geqq 1$ and $1 \leqq r \leqq+\infty, H^{s}\left(0, a ; x^{0}\right)$ is embedded continuously in $L^{r}(0, a) \cap$ $C^{s-1}((0, a])$. For $u \in H^{s}\left(0, a ; x^{0}\right)$

$$
|u|_{L^{r}\left(0, a ; x^{k}\right)} \leqq C|u|_{H^{s}\left(0, a ; x^{0}\right)}, \quad k \geqq 0,
$$

where $C$ depends on $r, a$ and is independent of $s$.

Proof. The first part is seen in [Ya1], Lemma 2.1. We prove the second part. First let $r=+\infty$. It follows from [A] that

$$
\sup _{x \in(0, a)} f(x)^{2} \leqq C\left(\int_{0}^{a} x f(x)^{2} d x+\int_{0}^{a} x f^{\prime}(x)^{2} d x\right),
$$

which implies that $H^{1}\left(0, a ; x^{0}\right)$ is continuously embedded in $L^{\infty}\left(0, a ; x^{0}\right)$. It is clear by the Sobolev lemma that $H^{s}\left(0, a ; x^{0}\right)$ is continuously embedded in $C^{s-1}((0, a])$. The case $r<+\infty$ is clear from $|f|_{L^{r}} \leqq C|f|_{L^{\infty}}$. (2.2) for $k>0$ follows from the inequality $|u|_{L^{r}\left(0, a ; x^{k}\right)} \leqq a^{k}|u|_{L^{r}\left(0, a ; x^{0}\right)}$

COROllary 2.1. Let $s \geqq 1$ and $k \geqq 0$. Let $1 \leqq r<+\infty$. For $u \in H^{s}\left(\Omega ; x^{\mu}\right)$ we have

$$
|u|_{L^{r}\left(\Omega ; x^{\mu+k}\right)} \leqq C|u|_{H^{s}\left(\Omega ; x^{\mu}\right)},
$$

where $C$ depends on $r, \mu, a$ and is independent of $s, T$. 
Lemma 2.1 ([Ya1]). For $u \in H_{0}^{1}\left(0, a ; x^{\mu}\right)$

$$
|u|_{L^{2}\left(0, a ; x^{\mu}\right)} \leqq a\left|\partial_{x} u\right|_{L^{2}\left(0, a ; x^{\mu+1}\right)} .
$$

This is the Poincaré type inequality in $H_{0}^{1}\left(0, a ; x^{\mu}\right)$.

2.2. Eigenvalue Problem for $L_{\mu}$. We shall consider the eigenvalue problem for $L_{\mu}$ :

$$
\left\{\begin{array}{l}
L_{\mu} \phi(x)=\lambda \phi(x), \quad x \in(0, a), \\
\phi(a)=0 .
\end{array}\right.
$$

Then we obtain the eigenvalues and the corresponding eigenfunctions (see [Ya1], section 3)

$$
\lambda_{j}=\frac{\mu_{j}^{2}}{4(\mu+1) a}, \quad \phi_{j}(x)=\frac{1}{a^{1 / 2} J_{\mu+1}\left(\mu_{j}\right)} \frac{J_{\mu}\left(\mu_{j} \sqrt{x / a}\right)}{x^{\mu / 2}},
$$

where $j \in N$. Here $\left\{\mu_{j}: j \in N\right\}$ is the set of all positive zero points of the $\mu$-order Bessel function $J_{\mu}(x)$ with

$$
\mu_{1}<\mu_{2}<\cdots
$$

For the Bessel functions and their zero points, see [T] and [W].

Proposition 2.3 ([Ya1]). $\left\{\phi_{j}\right\}$ is CONS in $L^{2}\left(0, a ; x^{\mu}\right)$ and a complete and orthogonal system in $H_{0}^{1}\left(0, a ; x^{\mu}\right)$.

\section{Formulation of Theorem}

In this section we shall consider IBVP

(P)

$$
\left\{\begin{array}{l}
\partial_{t}^{2} u(x, t)+L u(x, t)+f(x, u(x, t))=0, \quad(x, t) \in \Omega, \\
u(a, t)=0, \quad t \in(0, T), \\
u(x, 0)=\phi(x), \quad \partial_{t} u(x, 0)=\psi(x), \quad x \in(0, a),
\end{array}\right.
$$

and we shall show the existence of time-global weak solutions of $(\mathrm{P})$ that lie in the potential well. To formulate our theorem we shall define a weak solution and the potential well for $(\mathrm{P})$ and study its properties.

Weak Solutions. We define a weak solution of (P) as follows. Let $\phi \in H_{0}^{1}\left(0, a ; x^{0}\right)$ and $\psi \in L^{2}\left(0, a ; x^{0}\right)$. Let $Y$ be a space of test functions whose elements $\zeta$ belong to 
$H_{0}^{1}\left(\Omega ; x^{0}\right)$ and satisfy $\zeta(x, t)=0$ near $t=T$ for any $x \in(0, a)$. We call $u$ a weak solution of (P) if $u \in H_{0}^{1}\left(\Omega ; x^{0}\right)$ and the following identities hold:

$$
\begin{aligned}
& \int_{\Omega}\left(-\partial_{t} u(x, t) \partial_{t} \zeta(x, t)-x \partial_{x} u(x, t) \partial_{x} \zeta(x, t)\right. \\
& \quad+f(x, u(x, t)) \zeta(x, t)) d x d t \\
& =\int_{0}^{a} \psi(x) \zeta(x, 0) d x \\
& \int_{\Omega}\left(u(x, t) \partial_{t} \zeta(x, t)+\partial_{t} u(x, t) \zeta(x, t)\right) d x d t \\
& \quad=-\int_{0}^{a} \phi(x) \zeta(x, 0) d x
\end{aligned}
$$

for any $\zeta \in Y$. If $u$ is of $C^{2}$ and a weak solution of (P), then $u$ is the classical solution.

The Potential Well for (P). We denote the potential of $f$ by

$$
F(x, y)=\int_{0}^{y} f(x, \rho) d \rho .
$$

We shall define the potential energy $J(v)$ for $v \in H_{0}^{1}\left(0, a ; x^{0}\right)$ and the kinetic energy $K(w)$ for $w \in H_{0}^{1}\left(\Omega ; x^{0}\right)$ of our problem:

$$
J(v)=\int_{0}^{a}\left(\frac{1}{2} x v^{\prime}(x)^{2}+F(x, v(x))\right) d x
$$

for $v \in H_{0}^{1}\left(0, a ; x^{0}\right)$, and

$$
K(w)=\int_{0}^{a} \frac{1}{2}\left(\partial_{t} w(x, t)\right)^{2} d x
$$

for $w \in H_{0}^{1}\left(\Omega ; x^{0}\right)$. Clearly $J(0)=0$.

Let $\hat{E}(u, v)$ be denoted by

$$
\hat{E}(u, v)=\frac{1}{2} \int_{0}^{a} v(x)^{2} d x+J(u) .
$$

The total energy $E(t ; u)$ of the solution $u \in H_{0}^{1}\left(\Omega ; x^{0}\right)$ of $(\mathrm{P})$ is defined by

$$
E(t ; u)=\hat{E}\left(u(\cdot, t), \partial_{t} u(\cdot, t)\right)=K(u(\cdot, t))+J(u(\cdot, t)) .
$$

Proposition 3.1. Assume that $f(x, u)$ is continuous in $(x, u) \in[0, a] \times R_{u}^{1}$. Then $J(u)$ is continuous in $H_{0}^{1}\left(0, a ; x^{0}\right)$. 
Proof. Let $\left\{u_{j}\right\}$ be a sequence of $H_{0}^{1}\left(0, a ; x^{0}\right)$ tending to $u$ as $j \rightarrow+\infty$. Then it is clear that

$$
\left|\sqrt{x} \partial_{x}\left(u_{j}-u\right)\right|_{L^{2}\left(0, a ; x^{0}\right)} \rightarrow 0, \quad j \rightarrow+\infty .
$$

Next we see, by the mean value theorem,

$$
\int_{0}^{a}\left(F\left(x, u_{j}\right)-F(x, u)\right) d x=\int_{0}^{a} \int_{0}^{1} f\left(x, \theta u_{j}+(1-\theta) u\right)\left(u_{j}-u\right) d \theta d x .
$$

Since $u_{j}(x), u(x)$ are uniformly bounded in $(0, a)$ by Proposition 2.2 (ii), $f\left(x, \theta u_{j}+(1-\theta) u\right)$ is also uniformly bounded in $(0, a)$. From this and the Schwarz inequality we see

$$
\int_{0}^{a}\left(F\left(x, u_{j}\right)-F(x, u)\right) d x \rightarrow 0, \quad j \rightarrow+\infty .
$$

By (3.7) and (3.8) the conclusion holds.

We assume the following conditions on the outer force $f$ and its potential $F$.

(A) $f(x, u)$ is of $C^{1}$-class in $(x, u) \in[0, a] \times R_{u}^{1}$ and monotone decreasing in $u \in R^{1}$, and satisfies

$$
\begin{gathered}
-C_{0}|u|^{p+1} \leqq u f(x, u) \leqq-C|u|^{p+1}, \\
\gamma f(x, u) u-F(x, u) \leqq M
\end{gathered}
$$

for any $x \in[0, a]$ and $u \in R^{1}$. Here $\gamma \in(0,1 / 2)$ and $M, C_{0}, C>0$ are constants and $p>1$.

It follows from (A) that $f(x, u)$ has the zero point of $p$-order at $u=0$. We have $f$ and $F$ of the form

$$
f(x, u)=|u|^{p-1} u h(x, u), \quad F(x, u)=|u|^{p+1} H(x, u),
$$

where $p>1, h(x, u)$ and $H(x, u)$ are continuous in $u \in R_{u}^{1} \backslash\{0\}$ and satisfy $-C_{0} \leqq$ $h(x, u) \leqq-C$ and $-\frac{C_{0}}{p+1} \leqq H(x, u) \leqq-\frac{C}{p+1}$ in $[0, a] \times R_{u}^{1}$.

EXAMPLE 3.1. As a typical example of $f$ we take

$$
f(x, u)=-A(x) u^{3} .
$$

Here $A(x)$ is a positive $C^{1}$ function, and its derivative is bounded in $(0, a]$.

EXAMPLE 3.2. As another typical example of $f$ we take

$$
f(x, u)=-|u|^{p-1} u
$$

for $p>1$.

Lemma 3.1. Assume (A). Then there exists a neighborhood $V$ of $v=0$ such that $J(v) \geqq C|v|_{H^{1}\left(0, a ; x^{0}\right)}^{2}$ with a certain constant $C>0$ for all $v \in V$. 
PROOF. For brevity we write $|\cdot|_{H^{1}\left(0, a ; x^{0}\right)}$ by $|\cdot|_{H^{1}}$. Noting that $\sup _{x \in(0, a]}|v(x)| \leqq$ $C|v|_{H^{1}}$ by Proposition 2.2 (ii), we have

$$
\sup _{x \in(0, a]}|H(x, v(x))| \leqq M\left(|v|_{H^{1}}\right),
$$

where $M(s)$ is a continuous monotone increasing function of $s \in[0,+\infty)$ and hence, for $p>1$,

$$
\begin{aligned}
\int_{0}^{a}|F(x, v(x))| d x & =\int_{0}^{a}|v(x)|^{p+1}|H(x, v(x))| d x \\
& \leqq M\left(|v|_{H^{1}}\right) \int_{0}^{a}|v(x)|^{p+1} d x \\
& \leqq c M\left(|v|_{H^{1}}\right)|v|_{H^{1}}^{p+1},
\end{aligned}
$$

where $c$ is a positive constant. Here we have used Proposition 2.2 (ii) in the last stage. Using Lemma 2.1, we have

$$
J(v) \geqq \tilde{C}|v|_{H^{1}}^{2}-c M\left(|v|_{H^{1}}\right)|v|_{H^{1}}^{p+1} \geqq C|v|_{H^{1}}^{2},
$$

as long as $v \in V \equiv\left\{v ; c M|v|_{H^{1}}^{p-1}<\varepsilon\right\}$ for $p>1$ and $\varepsilon>0$ is suitably small. Thus the proof is complete.

LemmA 3.2. Assume (A). Let $u \in H_{0}^{1}\left(0, a ; x^{0}\right) \backslash\{0\}$. Then $J(\lambda u)$ is monotone increasing in $\lambda \geqq 0$ in a neighborhood of $\lambda=0$.

PROOF. It follows that

$$
\frac{d}{d \lambda} J(\lambda u)=\int_{0}^{a}\left(\lambda x u^{\prime}(x)^{2}+f(x, \lambda u(x)) u(x)\right) d x .
$$

Using (A), we can prove $\frac{d}{d \lambda} J(\lambda u)>0$ for small $\lambda>0$ by the similar argument as in the proof of Lemma 3.1. This shows the conclusion.

Now according to [Sat] with nonlinear wave equation, we shall define the potential well for (P) around the origin $u=0$. Let $u \neq 0$ in $H_{0}^{1}\left(0, a ; x^{0}\right)$ be fixed. Consider $J(\lambda u)$ as a function of $\lambda \geqq 0$. Then from (A), we have $J(\lambda u)$ is of $C^{1}$-class in $\lambda \geqq 0$ and from Lemma 3.2, $J(\lambda u)$ is monotone increasing in $\lambda$ in a neighborhood of $\lambda=0$. Let $\lambda=\lambda_{0}(u)>0$ be the first value of $\lambda$ at which $J(\lambda u)$ starts to decrease strictly. By (3.9) we have $\lambda_{0}(u)<+\infty$. Let us define

$$
d=\inf _{u \in H_{0}^{1}\left(0, a ; x^{0}\right) \backslash\{0\}} J\left(\lambda_{0}(u) u\right) .
$$

From (A), Lemmas 3.1 and 3.2 we see that $0<d<+\infty$. Let us define the potential well $W$ by

$$
W=\left\{u \in H_{0}^{1}\left(0, a ; x^{0}\right) ; 0 \leqq J(\lambda u)<d, 0 \leqq \lambda \leqq 1\right\}
$$


$d$ is called the depth of the potential well $W$.

PROPOSITION 3.2. W is bounded and open in $H_{0}^{1}\left(0, a ; x^{0}\right)$.

Proof. First we shall show that $W$ is bounded. The proof is done similarly to [Sat], p.161 and p.163. Let $u \in W$. Then $u$ satisfies

$$
J(u)=\int_{0}^{a}\left(\frac{1}{2} x u^{\prime}(x)^{2}+F(x, u(x))\right) d x<d .
$$

Since $J(\lambda u)$ is monotone increasing in $\lambda \in[0,1]$ from the definition of the potential well, we have

$$
\left.\frac{d}{d \lambda} J(\lambda u)\right|_{\lambda=1}=\int_{0}^{a}\left(x u^{\prime}(x)^{2}+f(x, u(x)) u(x)\right) d x \geqq 0 .
$$

Hence it follows from (3.15), (3.16) and (A) that

$$
\begin{aligned}
\frac{1}{2} \int_{0}^{a} x u^{\prime}(x)^{2} d x \leqq & -\int_{0}^{a} F(x, u(x)) d x \\
\leqq & +\int_{0}^{a}(\gamma f(x, u(x)) u(x)-F(x, u(x))) d x \\
& +\int_{0}^{a}(-\gamma f(x, u(x)) u(x)) d x \\
\leqq & +a M+\gamma \int_{0}^{a} x u^{\prime}(x)^{2} d x .
\end{aligned}
$$

Since $\gamma<1 / 2$, we have, by the Poincaré inequality in Lemma 2.1

$$
|u|_{H^{1}\left(0, a ; x^{0}\right)} \leqq M_{1},
$$

where $M_{1}$ is independent of $u$. This proves the boundedness of $W$.

Second we shall show that $W$ is open. Let $v_{0} \in W$ be arbitrarily fixed. Then we see that

$$
J\left(\lambda v_{0}\right)<d
$$

for $\lambda \in[0,1]$ and $J\left(\lambda v_{0}\right)$ is monotone increasing in $\lambda \in[0,1]$. We shall show that there exists an open ball $B\left(r ; v_{0}\right)$ with center $v_{0}$ and radius $r$ such that $B\left(r ; v_{0}\right) \subset W$. We prove this by contradiction. Assume the contrary. Then we can take $\left\{r_{j}\right\}$ tending to 0 as $j \rightarrow+\infty$ such that there exists $u_{j} \in B\left(r_{j} ; v_{0}\right)$ satisfying $u_{j} \notin W$. Then

$$
J\left(u_{j}\right) \geqq d .
$$

Since $\left\{u_{j}\right\}$ converges to $v_{0}$, we have, by the continuity of $J(u)$ in $u$,

$$
J\left(u_{j}\right) \rightarrow J\left(v_{0}\right), \quad j \rightarrow+\infty .
$$

Hence we get $J\left(v_{0}\right) \geqq d$. This is the contradiction. 
Main Theorem. We assume the following conditions on the initial data $\phi, \psi$. (B) Let $\phi \in W$ and $\psi \in L^{2}\left(0, a ; x^{0}\right)$. $\phi$ and $\psi$ satisfy the following condition

$$
\int_{0}^{a}\left\{\frac{1}{2}\left(\psi(x)^{2}+x \phi^{\prime}(x)^{2}\right)+F(x, \phi(x))\right\} d x<d .
$$

Our result reads as follows.

Main Theorem. Assume (A) and (B). Then IBVP (P) has a weak solution $u \in$ $H_{0}^{1}\left(\Omega ; x^{0}\right)$ satisfying $u(\cdot, t) \in W$ for all $t \in(0, T)$.

REMARK 3.1. Clearly the energy of the above solution $u$ is conserved

$$
E(t ; u)=E(0 ; u) \equiv \hat{E}(\phi, \psi)
$$

for $t \in(0, T)$.

\section{Proof of Main Theorem}

4.1. Approximate Solutions of $(\mathbf{P})$. We shall construct approximate solutions of $(\mathrm{P})$ by using the Galerkin method. Since $\phi \in H_{0}^{1}\left(0, a ; x^{0}\right)$ and $\psi \in L^{2}\left(0, a ; x^{0}\right)$, we can expand $\phi$ and $\psi$ into the Fourier series

$$
\begin{aligned}
& \phi=\sum_{i=1}^{\infty} a_{i} \phi_{i} \quad \text { in } \quad H_{0}^{1}\left(0, a ; x^{0}\right), \\
& \psi=\sum_{i=1}^{\infty} b_{i} \phi_{i} \quad \text { in } \quad L^{2}\left(0, a ; x^{0}\right),
\end{aligned}
$$

where $a_{i}=\left(\phi, \phi_{i}\right)$ and $b_{i}=\left(\psi, \phi_{i}\right), \quad i=1,2, \ldots$

We define a sequence of approximate solutions of the form

$$
u_{m}(x, t)=\sum_{i=1}^{m} a_{i}^{m}(t) \phi_{i}(x)
$$

that satisfy

$$
\left(\partial_{t}^{2} u_{m}+L u_{m}+f\left(x, u_{m}\right), \phi_{k}\right)=0, \quad k=1, \ldots, m
$$

with the initial condition

$$
u_{m}(\cdot, 0)=\alpha_{m}, \quad \partial_{t} u_{m}(\cdot, 0)=\beta_{m} .
$$

Here $\alpha_{m}(x)$ and $\beta_{m}(x)$ are of the form

$$
\alpha_{m}(x)=\sum_{i=1}^{m} a_{i} \phi_{i}(x), \quad \beta_{m}(x)=\sum_{i=1}^{m} b_{i} \phi_{i}(x) .
$$


(4.1)-(4.2) is IVP to a system of second-order ODEs with unknown functions $a_{1}^{m}(t), \ldots, a_{m}^{m}(t)$. Note that

$$
\left|\alpha_{m}-\phi\right|_{H^{1}\left(0, a ; x^{0}\right)} \rightarrow 0, \quad\left|\beta_{m}-\psi\right|_{L^{2}\left(0, a ; x^{0}\right)} \rightarrow 0
$$

as $m \rightarrow+\infty$.

PROPOSITION 4.1. Assume (A) and (B). Then there exists $m_{0} \in N$ such that for any $m \geqq m_{0}$ the following holds:

(i) IVP (4.1)-(4.2) has a unique solution $u_{m}(\cdot, t) \in C^{2}\left([0, T], H_{0}^{1}\left(0, a ; x^{0}\right)\right)$,

(ii) $u_{m}(\cdot, t) \in W$ for $t \in(0, T)$.

Proof. Since $f(x, u)$ is of $C^{1},\left(f\left(x, u_{m}\right), \phi_{k}\right)$ is locally Lipschitz continuous in $\left(a_{1}^{m}, \ldots, a_{m}^{m}\right)$. Therefore IVP (4.1)-(4.2) has a local solution in some interval [0, $\left.T_{0}\right)$.

We shall show that the energy of $u_{m}$ satisfies

$$
E\left(t ; u_{m}\right)<d
$$

for all $t \in\left(0, T_{0}\right)$ and sufficiently large $m$. Note that from Lemma $3.1 E\left(t ; u_{m}\right) \geqq 0$ in a neighborhood $V$ of $u=0$. Multiplying (4.1) by $\dot{a}_{k}^{m}$ and summing up from $k=1$ to $m$, we have

$$
\left(\partial_{t}^{2} u_{m}+L u_{m}+f\left(x, u_{m}\right), \partial_{t} u_{m}\right)=0,
$$

which implies that

$$
\frac{d}{d t} \int_{0}^{a}\left\{\frac{1}{2}\left(\partial_{t} u_{m}(x, t)^{2}+x\left(\partial_{x} u_{m}(x, t)\right)^{2}\right)+F\left(x, u_{m}(x, t)\right)\right\} d x=0 .
$$

Integrating this with respect to $t$ from 0 to $t$, we get

$$
\begin{array}{r}
\int_{0}^{a}\left\{\frac{1}{2}\left(\partial_{t} u_{m}(x, t)^{2}+x\left(\partial_{x} u_{m}(x, t)\right)^{2}\right)+F\left(x, u_{m}(x, t)\right)\right\} d x \\
=\int_{0}^{a}\left\{\frac{1}{2}\left(\beta_{m}(x)^{2}+x\left(\partial_{x} \alpha_{m}(x)\right)^{2}\right)+F\left(x, \alpha_{m}(x)\right)\right\} d x .
\end{array}
$$

Then, it follows from (3.18) in (B) and (4.4) that there exists $m_{0}$ such that for $m \geqq m_{0}$

$$
\int_{0}^{a}\left\{\frac{1}{2}\left(\beta_{m}(x)^{2}+x\left(\partial_{x} \alpha_{m}(x)\right)^{2}\right)+F\left(x, \alpha_{m}(x)\right)\right\} d x<d .
$$

Therefore we conclude from (4.6)-(4.7) that (4.5) is true.

We shall show that the solution exists globally in $t \in(0, T)$. We note that the initial data satisfies $E\left(0 ; u_{m}\right)<d$. Then let $T_{1}$ be the supremum of $t$ such that the solution $u_{m}(\cdot, t)$ exists for $t \in\left(0, T_{1}\right)$. We shall show that $T_{1}=T$. Assume the contrary i.e., $T_{1}<T$. Since $u_{m}\left(\cdot, \frac{T_{1}}{2}\right)$ satisfies (4.6), $u_{m}(\cdot, t)$ belongs to $W$ for all $t \in\left(0, \frac{T_{1}}{2}\right]$. Replacing the data by $\left\{u_{m}\left(\cdot, \frac{T_{1}}{2}\right), \partial_{t} u_{m}\left(\cdot, \frac{T_{1}}{2}\right)\right\}$, we conclude that the corresponding (approximated) solution $u_{m}(\cdot, t)$ can be extended into the interval $\left[\frac{T_{1}}{2}, T_{1}+\frac{T_{1}}{2}\right]$. Therefore the solution can be extended over 
$T_{1}$. Since $T_{1}$ is the supremum of the existence time, this shows the contradiction. Therefore $\operatorname{IVP}(4.1)-(4.2)$ has a global solution in $(0, T)$.

We shall show that $u_{m}(\cdot, t) \in W$ for $t \in(0, T)$. Note that $J\left(u_{m}(\cdot, t)\right)$ is continuous in $t$ by Proposition 3.1 and $\phi \in W$ by (B). Let $t_{1}$ be the supremum of $t$ such that $u_{m}(\cdot, t)$ belongs to $W$. Since $u_{m}(\cdot, 0)=\alpha_{m}$ belongs to $W$, we have $t_{1} \in(0, T]$. This shows $u_{m}(\cdot, t) \in W$ for all $t \in\left(0, t_{1}\right)$. We shall show $t_{1}=T$. Assume the contrary i.e., $t_{1}<T$. Since $W$ is open in $H_{0}^{1}\left(0, a ; x^{0}\right)$ by Proposition 3.2, we have $u_{m}\left(\cdot, t_{1}\right) \notin W$ by the continuity of $J$. Hence there exists $\lambda_{0} \in[0,1]$ such that $J\left(\lambda_{0} u_{m}\left(\cdot, t_{1}\right)\right) \geqq d$. On the other hand we have $\lim _{t \rightarrow t_{1}-0} J\left(\lambda_{0} u_{m}(\cdot, t)\right)=J\left(\lambda_{0} u_{m}\left(\cdot, t_{1}\right)\right)$, and since $J\left(\lambda u_{m}(\cdot, t)\right), t \in\left(0, t_{1}\right)$ is monotone increasing with respect to $\lambda$, we have $J\left(\lambda_{0} u_{m}(\cdot, t)\right) \leqq J\left(u_{m}(\cdot, t)\right)<d$. Combining these facts, we have

$$
\begin{aligned}
& J\left(\lambda_{0} u_{m}\left(\cdot, t_{1}\right)\right)=\lim _{t \rightarrow t_{1}-0} J\left(\lambda_{0} u_{m}(\cdot, t)\right) \\
& \quad \leqq \lim _{t \rightarrow t_{1}-0} J\left(u_{m}(\cdot, t)\right)=J\left(u_{m}\left(\cdot, t_{1}\right)\right)<d .
\end{aligned}
$$

Here we have the last inequality, since $J\left(u_{m}(\cdot, t)\right)<d$ holds for all $t \in(0, T)$ from (4.5). Thus the contradiction arises.

We shall proceed to extract a subsequence of $\left\{u_{m}\right\}$ that tends to the weak solution of $(\mathrm{P})$. We write the subsequence as $\left\{u_{m}\right\}$, taking subsequences step by step.

LEMMA 4.1. Assume (A) and (B). Then there exist a subsequence of $\left\{u_{m}\right\}$, again written as $\left\{u_{m}\right\}$, and $u \in H_{0}^{1}\left(\Omega ; x^{0}\right)$ such that

(i) $\left|u_{m}-u\right|_{L^{2}\left(\Omega ; x^{0}\right)} \rightarrow 0$,

(ii) $\left|\left(L u_{m}, \zeta\right)+\left(x \partial_{x} u, \partial_{x} \zeta\right)\right| \rightarrow 0$ for any $\zeta \in H_{0}^{1}\left(\Omega ; x^{0}\right)$ as $m \rightarrow+\infty$

Proof. Since $u_{m} \in W$ and satisfies (4.6), similarly to the proof of Proposition 3.2, we have

$$
\int_{0}^{a}\left(\left(\partial_{t} u_{m}(x, t)\right)^{2}+x\left(\partial_{x} u_{m}(x, t)\right)^{2}+u_{m}(x, t)^{2}\right) d x \leqq M_{1} .
$$

Integrating this inequality with respect to $t$ over $[0, T]$, we obtain

$$
\int_{\Omega}\left(\left(\partial_{t} u_{m}(x, t)\right)^{2}+x\left(\partial_{x} u_{m}(x, t)\right)^{2}+u_{m}(x, t)^{2}\right) d x d t \leqq M_{1} T .
$$

This means that $\left\{u_{m}\right\}$ is bounded in $H_{0}^{1}\left(\Omega ; x^{0}\right)$. Since every bounded set in $H_{0}^{1}\left(\Omega ; x^{0}\right)$ is compact in $L^{2}\left(\Omega ; x^{0}\right)$ by Proposition 4.1 in [Ya-Na-Ma], we see that there exists a subsequence of $\left\{u_{m}\right\}$, again written as $\left\{u_{m}\right\}$, and $u \in L^{2}\left(\Omega ; x^{0}\right)$ such that

$$
\left|u_{m}-u\right|_{L^{2}\left(\Omega ; x^{0}\right)} \rightarrow 0, \quad m \rightarrow+\infty .
$$

This proves the assertion (i). It follows from (4.8) that we can extract a subsequence of $\left\{u_{m}\right\}$ that converges weakly in $H_{0}^{1}\left(\Omega ; x^{0}\right)$. Therefore $u$ belongs to $H_{0}^{1}\left(\Omega ; x^{0}\right)$. 
In order to show the assertion (ii), we have only to note that $\left\{\sqrt{x} \partial_{x} u_{m}\right\}$ is bounded in $L^{2}\left(\Omega ; x^{0}\right)$. Hence $\left\{\left(L u_{m}, \zeta\right)\right\}$ is bounded so that by Proposition 2.1 (i), we can extract a subsequence of $\left\{\left(L u_{m}, \zeta\right)\right\}$ that converges to $-\left(x \partial_{x} u, \partial_{x} \zeta\right)$ for any $\zeta \in H_{0}^{1}\left(\Omega ; x^{0}\right)$.

4.2. Weak Solutions. We shall show that the function $u$ in Lemma 4.1 is a weak solution of (P). We first prove (3.1). Let $\xi(x, t)=\eta(t) \phi_{k}(x) \in H_{0}^{1}\left(\Omega ; x^{0}\right)$ for $k=1, \ldots, m$, where $\eta$ is of $C^{2}$ in $[0, T]$ and vanishes near a neighborhood of $t=T$. Then we see, from (4.1),

$$
\int_{\Omega}\left(\partial_{t}^{2} u_{m}+L u_{m}+f\left(x, u_{m}\right)\right) \xi d x d t=0 .
$$

Then we have, integrating by parts,

$$
\begin{aligned}
\int_{\Omega}( & \left.-\partial_{t} u_{m}(x, t) \partial_{t} \xi(x, t)+L u_{m}(x, t) \xi(x, t)\right) d x d t \\
& =\int_{0}^{a} \beta_{m}(x) \xi(x, 0) d x-\int_{\Omega} f\left(x, u_{m}(x, t)\right) \xi(x, t) d x d t .
\end{aligned}
$$

Letting $m \rightarrow+\infty$ in (4.9), we see from Lemma 4.1 that (3.1) holds for any $\xi$ above. In fact, Lemma 4.1 (ii) means

$$
\int_{\Omega} L u_{m} \xi d x d t \rightarrow-\int_{\Omega} x \partial_{x} u \partial_{x} \xi d x d t, \quad m \rightarrow+\infty .
$$

Integrating $\int_{\Omega} \partial_{t} u_{m}(x, t) \partial_{t} \xi(x, t) d x d t$ by parts in $t$, we have

$$
\begin{aligned}
\int_{\Omega} \partial_{t} & u_{m}(x, t) \partial_{t} \xi(x, t) d x d t \\
& =\int_{0}^{a} u_{m}(x, 0) \partial_{t} \xi(x, 0) d x-\int_{\Omega} u_{m}(x, t) \partial_{t}^{2} \xi(x, t) d x d t \\
& \rightarrow \int_{0}^{a} \phi(x) \partial_{t} \xi(x, 0) d x-\int_{\Omega} u(x, t) \partial_{t}^{2} \xi(x, t) d x d t \\
& =\int_{\Omega} \partial_{t} u(x, t) \partial_{t} \xi(x, t) d x d t
\end{aligned}
$$

We shall show

$$
\int_{\Omega} f\left(x, u_{m}\right) \xi d x d t \rightarrow \int_{\Omega} f(x, u) \xi d x d t .
$$

Since a subsequence of $\left\{u_{m}(x, t)\right\}$, written as $\left\{u_{m}(x, t)\right\}$ again, converges to $u(x, t)$ almost everywhere, $f\left(x, u_{m}(x, t)\right)$ also converges to $f(x, u(x, t))$ almost everywhere. By $u_{m} \in W$ in $t \in(0, T)$ we have, similarly to the proof of (3.17), $\left|u_{m}(\cdot, t)\right|_{H^{1}\left(0, a ; x^{0}\right)} \leqq M_{0}$ for $t \in(0, T)$, where $M_{0}$ is independent of $t$. Hence using Proposition 2.2 (ii), we have

$$
\left|u_{m}(x, t)\right| \leqq C\left|u_{m}(\cdot, t)\right|_{H^{1}\left(0, a ; x^{0}\right)} \leqq C M_{0}
$$


for any $(x, t) \in \Omega$. Therefore $\left\{f\left(x, u_{m}(x, t)\right)\right\}$ is uniformly bounded in $\Omega$. Hence using the Lebesgue bounded convergence theorem, we obtain (4.10). Thus, letting $m \rightarrow+\infty$ in (4.9) we have (3.1) for $\xi=\eta(t) \phi_{k}(x)$.

Let $Z$ be the set of the linear combinations $\sum_{k=1}^{l} \eta_{k}(t) \phi_{k}(x)$, where $\eta_{k}$ are of $C^{2}$ and vanish near a neighborhood of $t=T$. Then we have (3.1) for any $\xi \in Z$. Since $Z$ is dense in $Y$, we have (3.1) for $\zeta \in Y$. We can show (3.2) in the same way. Thus $u$ is the weak solution of $(\mathrm{P})$.

ACKNOWLEDGEMENTS. We would like to express our sincere gratitude to Professor Tokio Matsuyama for his valuable advices and comments and we would also like to thank the referee for his kind comments of the manuscript.

\section{References}

[A] R. A. Adams, Sobolev Spaces, Academic Press, 1975.

[Ko] B. G. Korenev, Bessel Functions and their Applications, Taylor and Francis Inc., 2002.

[K-G-S] N. S. Koshlyakov, E. V. Gliner and M. M. Smirnov, Differential Equations of Mathematical Physics, Moscow, 1962 (in Russian).

[L] J. L. Lions, Quelques Méthodes de Résolution des Problèmes aux Limites non linéaires, Paris, Dunod, 1969.

[Sat] D. H. SATtinger, On global solution of nonlinear hyperbolic equations, Arch. Rat. Mech. Anal. 30 (1968), 148-172.

[T] C. J. Tranter, Bessel Functions with Some Physical Applications, Hart Publishing Co., New York, 1969.

[W] G. N. Watson, Theory of Bessel Functions, Cambridge University Press, 1962.

[Ya1] M. YAMAGUCHI, Almost periodic oscillations of suspended string under quasiperiodic linear force, J. Math. Anal. Appl., 303, no. 2 (2005), 643-660.

[Ya2] M. YAMAGUCHI, Free vibrations of nonlinear equation of suspended string, preprint.

[Ya-Na-Ma] M. YAmaguchi, T. NAgAi and K. Matsukane, Forced oscillations of nonlinear damped equation of suspended string, preprint.

Present Addresses:

JAIPONG WONGSAWASDI

DEPARTMENT OF MATHEMATICS, FACULTY OF SCIENCE,

King MONGKUT'S InstituTE OF TECHNOLOGY LADKRABANG,

CHALONGKRUNG LADKRABANG, BANGKOK 10520, THAILAND.

e-mail: kwjaipon@kmitl.ac.th

MASARU YAMAGUCHI

Department of MATHEMATics, FACUlty of SCIENCE,

TOKAI UNIVERSITY,

HIRATSUKA, KANAGAWA, 259-1292 JAPAN.

e-mail: yamagchi@keyaki.cc.u-tokai.ac.jp 